\title{
Electron Cooling against IBS for High Energy Colliders
}

\author{
A. Burov, FNAL
}

\begin{abstract}
Analytical formulas for detailed longitudinal IBS and electron cooling rates are presented for bunched beams. Ratio of electrons over ions (pbars) per bunch required for IBS compensation is found.
\end{abstract}

Originally, magnetized cooling force has been calculated by Ya. Derbenev [1]; he considered the electron beam as a dielectric medium. Later, V. Parkhomchuk suggested another derivation, based on a binary collision model, and got a different result [2]. This difference between the two approaches was understood by V. Lebedev [3], who pointed out that the dielectric model cannot be applied at small distances, which still contribute to the Coulomb logarithm; thus, the model gives only a part of the force. From another hand, the binary collision approach is valid for the whole range of the impact parameters contributing to the logarithm; thus, it is correct with the logarithmic accuracy. Binary collision model gives the cooling force which in the beam frame can be expressed as

$$
\vec{F}=-P \overrightarrow{\mathbf{v}} / \mathbf{v}-\boldsymbol{Q} \overrightarrow{\boldsymbol{b}}(\overrightarrow{\mathbf{v}} \overrightarrow{\boldsymbol{b}}) / \mathbf{v}
$$

where $\overrightarrow{\boldsymbol{b}}$ is a unit vector along magnetic field, $\overrightarrow{\mathrm{V}}$ is the ion velocity,

$$
\boldsymbol{P}=\alpha\left(1-2(\overrightarrow{\mathrm{v}} \vec{b})^{2} / \mathbf{v}^{2}\right) ; \quad \boldsymbol{Q}=\alpha ; \quad \alpha \equiv \frac{2 \pi n_{e} e^{4} L_{e}}{m \mathbf{v}^{2}}
$$

For non-magnetized cooling, $\boldsymbol{P}=\mathbf{2} \alpha ; \boldsymbol{Q}=\mathbf{0}$.

Magnetized electron cooling rate for longitudinal degree of freedom of a bunched beam is obtained from the cooling force by averaging over the three phases of the circulating ion, leading to (laboratory frame)

$$
\Lambda_{e}^{\|} \equiv-\frac{1}{J_{z}} \frac{d J_{z}}{d t}=\frac{8 N_{e} r_{e} r_{i} c \eta_{c} L_{e}}{\gamma^{2} z_{m}}\left\langle\frac{n_{\perp}}{\mathrm{v}^{3}} \frac{\mathrm{v}_{\perp}^{2}}{\mathrm{v}^{2}}\right\rangle_{x, y}
$$

Here $\boldsymbol{z}_{\boldsymbol{m}}$ is the ion amplitude in the longitudinal direction, $\boldsymbol{J}_{z}=\boldsymbol{\omega}_{s} \boldsymbol{z}_{\boldsymbol{m}}^{2} / \mathbf{2}$ is the longitudinal action, and the brackets $\langle\ldots\rangle_{x, y}$ stand for the transverse $(\boldsymbol{x}, \boldsymbol{y})$ phase averaging (the longitudinal averaging is already done here, assuming the e-bunch is short). This double integral is simplified when the longitudinal beam temperature is small compared with the transverse. In this case, assuming the Gaussian shape of the e-bunch 
with rms size $\sigma_{e}$, a negligible longitudinal temperature and transverse electron rms velocity $\boldsymbol{u}_{\boldsymbol{e}}$ (all the velocities are in units of the speed of light $\boldsymbol{c}$ ), the rate writes as

$$
\Lambda_{e}^{\|}=\frac{8 N_{e} r_{e} r_{i} \eta_{c} L_{e}}{3 \pi \gamma^{2} z_{m} \mathbf{v}_{z m} u_{e}^{2} \sigma_{e}^{2}} \exp \left(-\frac{x_{m}^{2}+y_{m}^{2}}{4 \sigma_{e}^{2}}-\frac{\mathbf{v}_{x m}^{2}+\mathbf{v}_{y m}^{2}}{4 u_{e}^{2}}\right) I_{0}\left(\frac{x_{m}^{2}}{4 \sigma_{e}^{2}}-\frac{\mathbf{v}_{x m}^{2}}{4 u_{e}^{2}}\right) I_{0}\left(\frac{y_{m}^{2}}{4 \sigma_{e}^{2}}-\frac{\mathbf{v}_{y m}^{2}}{4 u_{e}^{2}}\right)
$$

where subscripts $m$ point to amplitude values of the offsets and velocities in the beam frame, and $I_{0}(x)$ is the modified Bessel function. The transverse e-cooling rates are by a factor of $\cong \mathbf{v}_{z m} / \mathbf{v}_{x m}$ smaller than the longitudinal one. The rate for non-magnetized cooling is expressed by the same formula, with only difference that the numerical factor is $\mathbf{4}$ instead of $\mathbf{8} / \mathbf{3}$.

The longitudinal IBS rate is calculated in a similar way. For the longitudinally cold and transversely round beam, the longitudinal IBS diffusion can be presented as

$$
\begin{aligned}
& D_{i}^{\|} \equiv \frac{d}{d t} \mathbf{v}_{z m}^{2}=\frac{4 \pi N_{i} r_{i}^{2} c L_{i}}{\gamma^{2}}\left\langle\frac{n}{\Delta \mathbf{v}_{\perp}}\right\rangle_{x, y, z}= \\
& \sqrt{\frac{2}{\pi}} \frac{N_{i} r_{i}^{2} c L_{i}}{\gamma^{2} \mathbf{v}_{z m}^{2} \sigma_{x}^{2} \sigma_{z} u_{x}} \exp \left(-\frac{z_{m}^{2}}{4 \sigma_{z}^{2}}\right) I_{0}\left(\frac{z_{m}^{2}}{4 \sigma_{z}^{2}}\right) \mathcal{g}\left(\frac{\mathbf{v}_{x m}}{\boldsymbol{u}_{x}}, \frac{\mathbf{v}_{y m}}{u_{x}}\right)
\end{aligned}
$$

with

$$
\mathcal{G}(x, y) \equiv \frac{2}{\pi} \exp \left(-\frac{x^{2}+y^{2}}{2}\right) \int_{0}^{\infty} \int_{0}^{\infty} \frac{d \xi d \eta}{\sqrt{\xi^{2}+\eta^{2}}} \exp \left(-\frac{\xi^{2}+\eta^{2}}{2}\right) I_{0}(\xi x) I_{0}(\eta y) .
$$

The symbols $\boldsymbol{u}_{x, y}$ are rms velocities in the beam frame and $\sigma_{x, y}$ are the rms beam sizes.

Note that this expression gives a detailed diffusion, i. e.. the diffusion as a function of all the three amplitudes. After averaging over Gaussian distribution, it leads to the conventionally used beam-averaged value:

$$
\frac{d}{d t} \overline{\mathbf{v}_{z}^{2}}=\frac{N_{i} r_{i}^{2} c L_{i}}{8 \gamma^{2} \sigma_{x}^{2} \sigma_{z} u_{x}}
$$

These IBS formulas assume longitudinally cold beam, $\mathbf{v}_{z m} / \mathbf{v}_{x m}<<1$. When this ratio is not extremely small, a correction due to its final value can be significant. It can be shown, that this correction of the longitudinal IBS diffusion can be approximated by a factor of $1-\sqrt{\mathbf{v}_{z m} /\left(\boldsymbol{u}_{x} \sqrt{\mathbf{2}}\right)}$, to be consistent with [4] for any ratio of velocities. After that, the longitudinal IBS rate is presented as 


$$
\Lambda_{i}^{\|} \equiv \frac{1}{\mathbf{v}_{z m}^{2}} \frac{d}{d t} \mathbf{v}_{z m}^{2}=\sqrt{\frac{2}{\pi}} \frac{N_{i} r_{i}^{2} c L_{i}}{\gamma^{2} \mathbf{v}_{z m}^{2} \sigma_{x}^{2} \sigma_{z} u_{x}} \exp \left(-\frac{z_{m}^{2}}{4 \sigma_{z}^{2}}\right) I_{0}\left(\frac{z_{m}^{2}}{4 \sigma_{z}^{2}}\right) G\left(\frac{\mathbf{v}_{x m}}{u_{x}}, \frac{\mathbf{v}_{y m}}{u_{x}}\right)\left(1-\sqrt{\frac{\mathbf{v}_{z m}}{u_{x} \sqrt{2}}}\right)
$$

Horizontal IBS rate $\Lambda_{i}^{x} \equiv \boldsymbol{d}\left(\ln J_{x}\right) / d t$ relates to the longitudinal one as

$$
\Lambda_{i}^{x} / \Lambda_{i}^{\|}=\frac{\overline{D^{2}+\left(D^{\prime} \beta_{x}+\alpha_{x} D\right)^{2}}}{x_{m}^{2}} \mathbf{v}_{z m}^{2}
$$

where the bar stands for the orbit-averaging.

Below, the plot for electron cooling and IBS longitudinal rates versus transverse actions is presented for $\boldsymbol{N}_{\boldsymbol{i}}=\mathbf{1 \cdot 1 0 ^ { 9 }}$ Au ions in RHIC at $\gamma=\mathbf{1 0 0}$ with normalized $95 \%$ emittances $\varepsilon_{n x}=\varepsilon_{n y}=15 \mathrm{~mm}$ mrad, rms momentum spread $\mathbf{8 . 5} \cdot \mathbf{1 0}^{-4}$, rms bunch length $30 \mathrm{~cm}$.

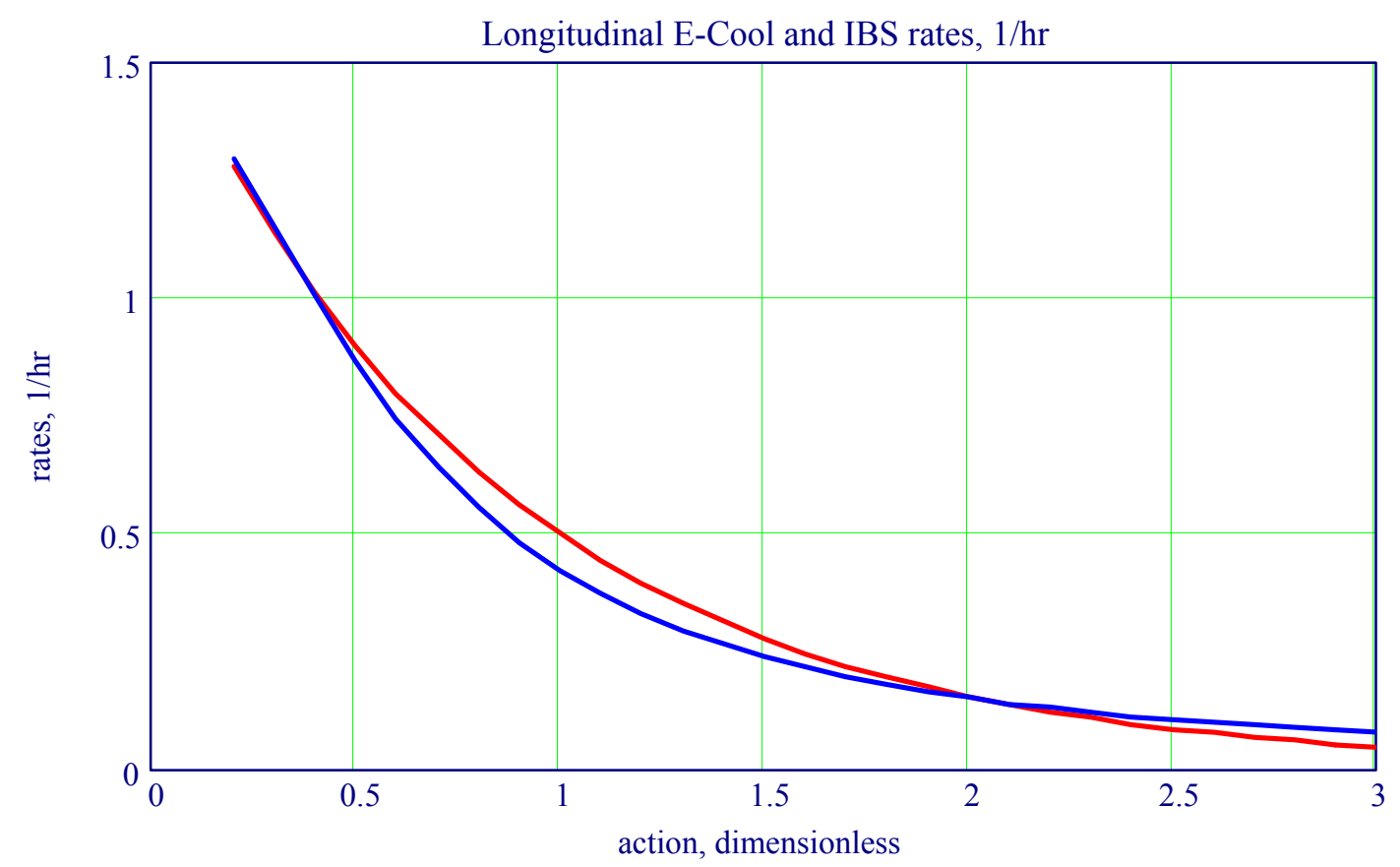

Fig. 1. Longitudinal IBS (blue line) and e-cool (red line) rates for Au ions in RHIC for $N_{e}=\mathbf{6} \cdot 10^{10}, \sigma_{e}=1.3 \sigma_{x}, \boldsymbol{u}_{e}=\mathbf{1 . 3} \boldsymbol{u}_{x}$, Coulomb $\operatorname{logs} \boldsymbol{L}_{e}=\mathbf{2 . 5}$, $\boldsymbol{L}_{i}=\mathbf{1 9}$, cooler's fraction $\eta_{c}=\mathbf{7 . 8} \cdot \mathbf{1 0}^{-3}$, and the beta-function in the cooler $\beta_{c}=60 \mathrm{~m}$. Both transverse actions are equal for this plot, and measured in units of the rms emittance. The longitudinal action is equal to the longitudinal rms emittance.

The result obtained can be expressed in terms of the number of electrons per bunch required to compensate longitudinal IBS of the given number of ions per bunch, as it was suggested in [5]. Assuming that the 6D phase space shape of the electron bunch is 
optimized, it comes out that the number of electrons required for longitudinal IBS compensation is

$$
\frac{N_{e}}{N_{i}}=f \frac{L_{i}}{L_{e}} \frac{r_{i}}{r_{e}} \frac{1}{\eta_{c}} \frac{u_{x}}{u_{z}}\left(1-\sqrt{\frac{u_{z}}{u_{x}}}\right),
$$

where a numerical factor $f=\mathbf{2}$ for magnetized cooling and $f=\mathbf{4 / 3}$ for nonmagnetized.

Note that the electron beam transverse size is optimized to match e-cool and IBS rates. It follows from this plot that $N_{e}=\mathbf{6} \cdot \mathbf{1 0}^{\mathbf{1 0}}$ is required to compensate longitudinal IBS growth of $\boldsymbol{N}_{\boldsymbol{i}}=\mathbf{1} \cdot \mathbf{1 0}^{9}$ gold ions. However, the horizontal IBS growth, whose rate is about equal to the longitudinal one, is not compensated by the transverse component of the cooling force (1), which is too small and even has a wrong sign. To compensate horizontal IBS diffusion, dispersive electron cooling scheme can be applied, which requires higher number of electrons, $\boldsymbol{N}_{e} \geq \mathbf{2} \cdot \mathbf{1 0}^{11}$. So high number of electrons (positrons) per bunch could be accumulated in a storage ring, where required emittances and energy spread could be obtained by means of a wiggler.

For Tevatron at full energy, the longitudinal IBS compensation would require $\boldsymbol{N}_{e} / \boldsymbol{N}_{i}=\mathbf{3 0}$ electrons over protons per bunch ratio (non-magnetized cooling), assuming $20 \mathrm{~m}$ of the cooling length and matched e-bunch. Taking $N_{i}=\mathbf{3} \cdot \mathbf{1 0}^{11}$, it leads to $N_{e}=1 \cdot 10^{13}$. So high bunch population means that the storage ring cooler has to be considered, where the energy spread will be at least 5-10 times higher than protons one [6]. Horizontal IBS compensation requires to increase this number by a factor of 4 or so, totally leading to $N_{e}=(\mathbf{2}-\mathbf{4}) \cdot \mathbf{1 0}^{14}$ electrons, which is about 2 orders of magnitude above possibilities.

\section{References}

[1] Y. S. Derbenev, A. N. Skrinsky, Sov. Plasma Phys., Vol. 4, 3, p.492 (1978).

[2] V. V. Parkhomchuk, "Physics of Fast Electron Cooling", Proc. of Workshop on Electron Cooling and Related Applications, Karlsruhe (1984).

[3] N. Dikansky et al, "Ultimate Possibilities of Electron Cooling", Budker INP Preprint 88-61, Novosibirsk, 1988.

[4] J. D. Bjorken, S. K. Mtingwa, Part. Acc. Vol. 13, p. 115 (1983).

[5] Y. Derbenev, in Proc. EPAC 2000.

[6] P. Ivanov, unpublished note, Jan. 2003. 
